\title{
Mycelium development in Streptomyces antibioticus ATCCI I 89 I occurs in an orderly pattern which determines multiphase growth curves
}

\author{
Angel Manteca1, Marisol Fernandez ${ }^{2}$ and Jesus Sanchez ${ }^{* 1}$
}

Address: ${ }^{1}$ Universidad de Oviedo, Facultad de Medicina, Area de Microbiologia, Departamento de Biologia Funcional, 33006, Julian Claveria s/n, Oviedo, Spain and 2Laboratorio de Proteomica, Centro Nacional de Biotecnologia, Cantoblanco, 28049 Madrid, Spain

Email: Angel Manteca - mantecaangel@uniovi.es; Marisol Fernandez - msfdez@cnb.uam.es; Jesus Sanchez* - jsm@fq.uniovi.es

* Corresponding author

Published: 15 September 2005

BMC Microbiology 2005, 5:5 I doi:|0.||86/|47|-2|80-5-5|
Received: 3I May 2005

Accepted: 15 September 2005

This article is available from: http://www.biomedcentral.com/147|-2180/5/5 I

(C) 2005 Manteca et al; licensee BioMed Central Ltd.

This is an Open Access article distributed under the terms of the Creative Commons Attribution License (http://creativecommons.org/licenses/by/2.0), which permits unrestricted use, distribution, and reproduction in any medium, provided the original work is properly cited.

\begin{abstract}
Background: The current model for the developmental cycle of Streptomyces confluent cultures on agar surface is based on the assumption that the only differentiation takes place along the transverse axis (bottom-up): a vegetative (substrate) mycelium grows completely live and viable on the surface and inside the agar until it undergoes a death process and differentiates to a reproductive (aerial) mycelium which grows into the air. Hence, this vertical description assumes that the development in the pre-sporulating phases is more or less homogeneous in all zones of the plate surface.

Results: The work presents a detailed analysis of the differentiation cycle in Streptomyces antibioticus ATCCII89I considering a different spatial dimension: the longitudinal axes, represented by the plate surface. A previously unsuspected complexity during the substrate mycelial phase was detected. We have demonstrated that the young substrate hyphae suffer an early death round that has not been previously described. Subsequently, the remaining mycelium grows in successive waves which vary according to the density of the spore inoculum. In the presence of dense inocula ( $1.5 \times 10^{6}$ spores per plate), the hyphae develop in regular circles, approximately $0.5 \mathrm{~cm}$ in diameter. By contrast, with highly diluted inocula $\left(6 \times 10^{3}\right.$ spores per plate), aerial mycelium develops initially in the form of islands measuring $0.9 \mathrm{~mm}$ in diameter. Further mycelial development occurs between the circles or islands until the plate surface is totally covered. This pattern persists throughout the entire developmental cycle including the sporulation phases.
\end{abstract}

Conclusion: An early death round during the substrate mycelial phase of Streptomyces antibioticus ATCCII89I takes place prior to successive growth periods in surface cultures. These developmental periods in turn, determine the shape of the complex multiphase growth curves observed. As shown here, these results also apply to other Streptomyces strains and species. Understanding these peculiarities of the Streptomyces developmental cycle is essential in order to properly interpret the morphological/biochemical data obtained from solid cultures and will expand the number of potential phenotypes subject to study. 


\section{Background}

Streptomyces is a naturally occurring bacterium in soil and is likely to be present in aquatic habitats as well [1]. Since the early discovery of this microorganism's ability to to produce clinically useful antibiotics [2,3], the bacterium has received tremendous scientific attention [4]. Furthermore, other noteworthy characteristics, such as its remarkably complex developmental features, make this microorganism an interesting subject of study. Early on, Streptomyces was seen to form two distinct structures when grown on culture surfaces [5]: a substrate (vegetative) mycelium and an aerial (reproductive) mycelium. Substrate mycelium, which is assumed to grow into the medium, has a mean diameter of $0.7 \mu \mathrm{m}$ and is bound by a 0.01-0.02 $\mu \mathrm{m}$ thick mucopeptide cell wall (reviewed in $6)$. This mycelium is assumed to be present in different stages of cellular degeneration during all growth phases. Early reports stated that aerial hyphae were the result of simple branching of substrate hyphae [7] and were preceded by a short period of decreased macromolecular synthesis [8]. One important feature of the aerial hyphae is that their outer surface is covered with a superficial fibrous sheath [7,9-11]. All these reports described the Streptomyces life cycle as a bottom-up (substrate-aerial) process. Consequently, it was assumed that development was uniform throughout the entire plate surface.

Our previous works have presented a detailed analysis of S. antibioticus development [12-14]. To obtain a reliable picture of the cell death phenomena that accompany this process, we have used a technique to analyse bacterial viability that involves staining the nucleic acids of the damaged (leaky) cells with propidium iodide (PI) [12]. This dye only enters cells with damaged membranes and substantially enhances fluorescence by binding to nucleic acids with little or no sequence preference (references in 15). PI staining, alone or in combination with fluorescein derivatives, has been widely used for cell death analysis in bacteria [16-19] and also in eukaryotic cells [20]. The reliability of this method has been also assessed in Streptomyces in submerged $[13,21]$ and surface $[12,14]$ conditions. Here we have extended our studies to a third dimension: the longitudinal axes on the plate surface. As illustrated below, this perspective is fundamental to understanding the developmental cycle of this bacterium.

\section{Results}

Confocal laser-scanning fluorescence microscopy (CLSM) analysis of development-linked cell death processes of Streptomyces antibioticus ATCCI I89 I in confluent surface cultures

Figure 1 presents a global perspective of some of the most relevant features of the different developmental steps analysed in Streptomyces antibioticus ATCC11891 on surface
GAE cultures. To facilitate a sequential view of the process, we have divided it into several phases (A-H; Figure 1).

Phase A (0-7 hours) consists of germination and early hyphae development. When the spores are spread out on the surface (normally with a bent glass stick), all of them are viable (stained green) and remain in relatively large groups, probably owing to their hydrophobic properties (not shown). Germination begins at 4 hours by the asynchronous emission of a single (or less frequently, double) germ tube (Figure 1A, 5 and 6 hours). Most of these young hyphae undergo a very early death process (Figure 1A, 5 and 6 hours), which is remarkably symmetrical in a large proportion of the cases: live and dead segments alternate in a highly regular fashion within the same hyphae (Figure 1A, 6 hours); we have named them variegated hyphae [14]. This death process affects most of the young hyphae, although a small proportion of spores emit a germ tube that, while it is initially totally viable (Figure 1A, 6 hours), will eventually die. This is not unexpected, given that the spores are not distributed homogeneously throughout the plate and the microenvironment encountered by each of them may differ.

In Phase B (7-10 hours), the plate is completely covered with a thin layer of variegated mycelium (Figure $1 \mathrm{~B}_{1}, 8$ hours). At this stage, the appearance of thin, green rings (Figure $1 \mathrm{~B}_{1}, 8$ hours) measuring approximately $0.5 \mathrm{~cm}$ in diameter is particularly noteworthy. These rings are formed by the hyphae of late-germinating spores that remain live (high magnification in figure $1 \mathrm{~B}_{2}, 8$ hours), and contrast with the mycelium originating from the previously germinated spores; hence, the variegated appearance. The center of the circle delimitated by the ring, as well as the mycelium located between the rings, is made up of variegated hyphae.

During Phase C (10-14 hours), the young, non-variegated hyphae referred to in Phase $\mathrm{B}$ that comprise the border of the circles undergo rapid, profuse growth (Figure 1C, 11 hours).

Phase D (14-16 hours) shows an overall decline in the grow rate (see below, Figure $3 \mathrm{~A}$ ). The borders of the circles cease to grow and live mycelium begins to slowly develop in the center (Figure 1D, 15 hours). This mycelium differs from the live mycelium in the borders of the circles in that it originates from the extension of the viable segments within the variegated hyphae (Figure 1A, 6 hours) and not from late-germinating spores, as occurs on the outer surface of the circles (see above and Discussion). The mycelium develops in the form of islands (Figure 1D, 15 hours), which appear near the edge of the circles and spread in radial waves towards the center (Figures $1 D_{1}-D_{3}$, 15 hours). This radial pattern of growth from the edge of 

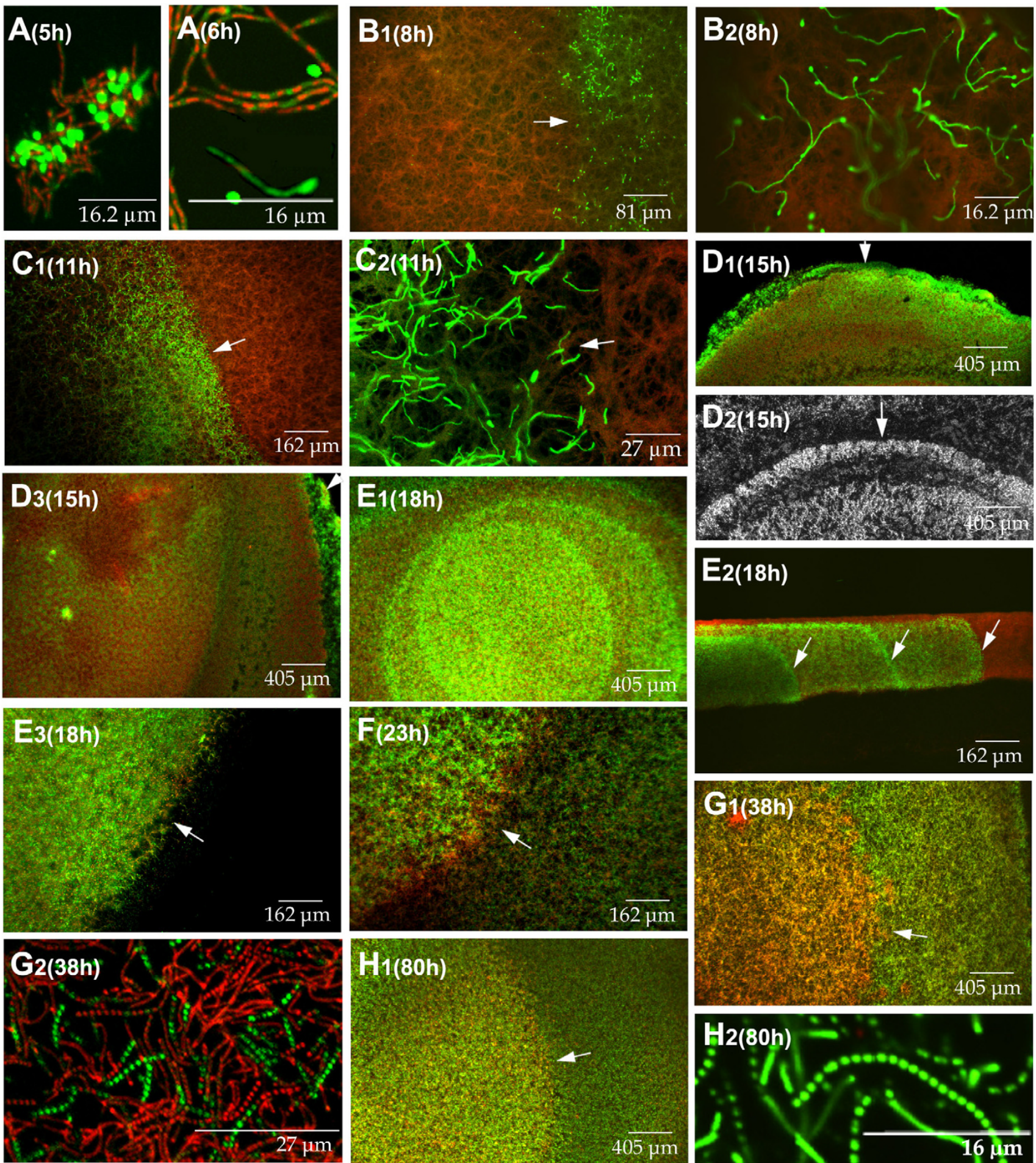

\section{Figure I}

Confocal laser-scanning fluorescence microscopy analysis of the development-linked cell death processes of Streptomyces antibioticus ATCCI I89I in confluent surface cultures. Developmental phases (A-H) and culture times (hours) are indicated. Picture $D_{2}$ was obtained under the phase contrast microscope. The other images correspond to culture sections stained with SYTO 9 and propidium iodide. $E_{2}$ is a cross section view; the other images correspond to longitudinal sections (see methods). Arrows in $E_{2}$ indicate the eccentric circles of live mycelium developing from the bottom upwards, forming distinct layers with welldefined boundaries. Arrows in the rest of the images indicate circle edges. For details see text. 

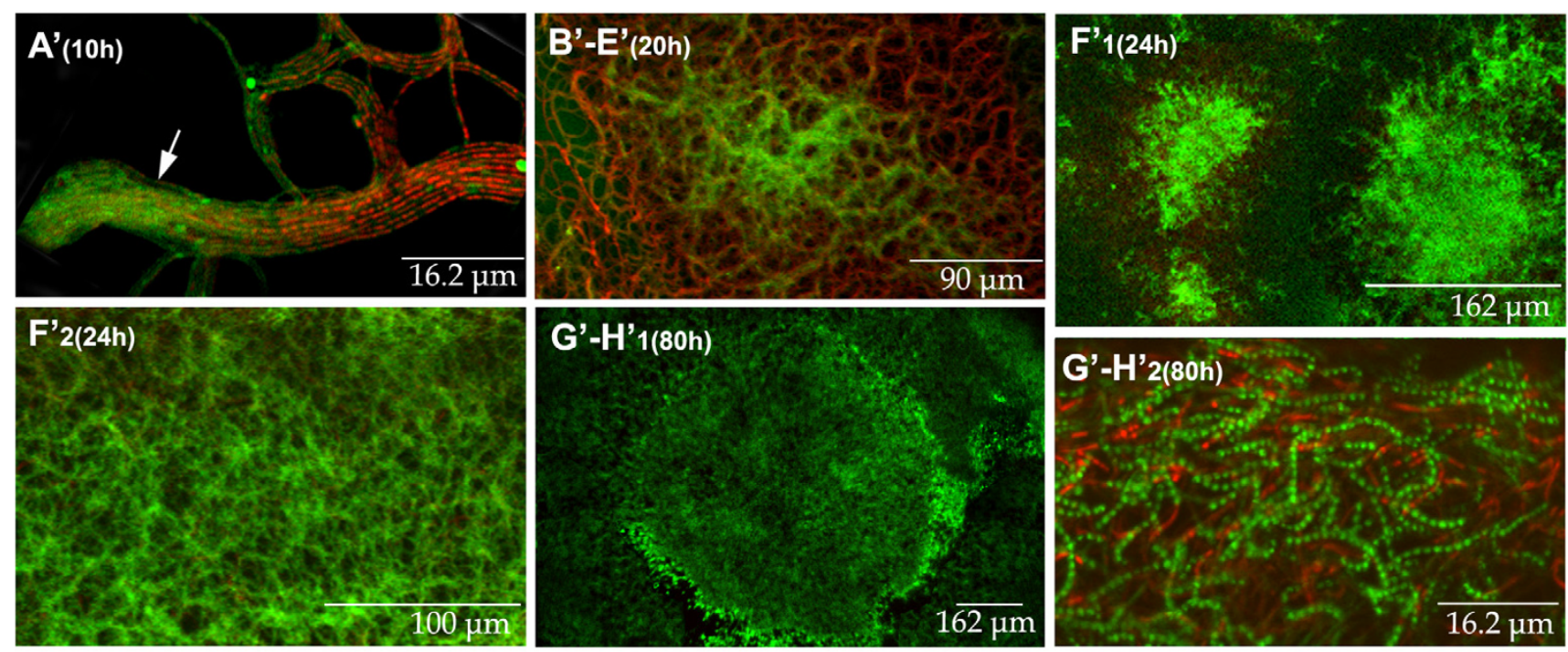

Figure 2

Longitudinal sections of Streptomyces antibioticus ATCCI I89I surface cultures obtained using a diluted inoculum $\left(6 \times 10^{3}\right.$ spores per plate). The developmental phases $\left(\mathrm{A}^{\prime}-\mathrm{H}^{\prime}\right)$ and culture times are indicated. Samples were stained with SYTO 9 and propidium iodide. Arrow in picture $A^{\prime}$ indicates a group of hyphae in transition from presenting uniform green fluorescence to a variegated appearance, in which live (green) and dead (red) segments alternate in the same hypha. See text for details.

the circles inwards leaves clear areas near the center, unoccupied by islands (Figure $1 \mathrm{D}_{3}, 15$ hours). Hence, at this point the plate is uniformly covered with mycelium arranged in dense circles separated by less dense areas. Figures $1 \mathrm{D}_{1}$ and $1 \mathrm{D}_{2}$ (15 hours) show a mycelial circle in Phase $\mathrm{D}$; arrows indicate the edge of the circle as observed under CLSM (Figure $1 \mathrm{D}_{1}$ ) and under non-fluorescence, phase-contrast microscopy (Figure $1 \mathrm{D}_{2}$ ). The mycelium outside the circle is evident in the latter. This mycelium does not reveal fluorescence in Figure $1 \mathrm{D}_{1}$ at the magnification used, due to its lower density. The circles are distributed quite symmetrically (approximately one per 2.5 $\mathrm{cm}^{2}$ ), as deduced from the microscopic analyses (not shown in the pictures).

During Phase E (16-20 hours), there is profuse growth of the live mycelial islands located in the center of the circles described above. Occasionally, several eccentric circles can be seen in the center of the largest circle (Figure $1 \mathrm{E}_{1}$, 18 hours). Figure $1 \mathrm{E}_{2}$ shows a cross section of the mycelial layer during Phase E: the eccentric circles of live mycelium develop from the bottom up, forming separate layers with well defined boundaries (arrows in Figure $1 \mathrm{E}_{2}$ ). Figure $1 \mathrm{E}_{3}$ shows the edge of a circle during this phase. No fluorescence is observed outside the circle, owing to the lower density of mycelium located there (see also Figures $1 D_{1}$ and $1 \mathrm{D}_{2}$ ).
Phase F (20-30 hours) is characterized by the mycelial growth between the circles described in the earlier phases (Figure 1F, 23 hours). These areas remain less dense than the circles and are made up of live hyphae.

Phase G (30-45 hours) represents the culmination of the second death round in the substrate mycelium, as well as the pre-sporulating areas of aerial mycelium [6,22-25]; Figure $1 \mathrm{G}, 38$ hours]. The majority of the hyphae located in the center of the circles are dead and present segmented DNA in the nucleoids (Figure $1 G_{2}, 38$ hours).

Phase $\mathrm{H}$ (45-96 hours) is the sporulation phase. Live hyphae grow and form spores inside and outside the circles (Figure $1 \mathrm{H}_{1}, 80$ hours). The mycelium between the circles has also suffered a second death round prior to sporulation, although it is less extensive than the one that takes place inside the circles and is not visible at the magnification shown. The change in colour (arrow in Figure $1 \mathrm{H}_{1}, 80$ hours) indicates the border of the original circle, which continues to reveal greater density than the areas between circles. Figure $1 \mathrm{H}_{2}$ ( 80 hours) shows a detail of the viable chains of spores.

The result of this entire process is a variability in the developmental phases across the plane of a solid agar surface. In this work will refer to this phenomenon as "longitudinal heterogeneity". 

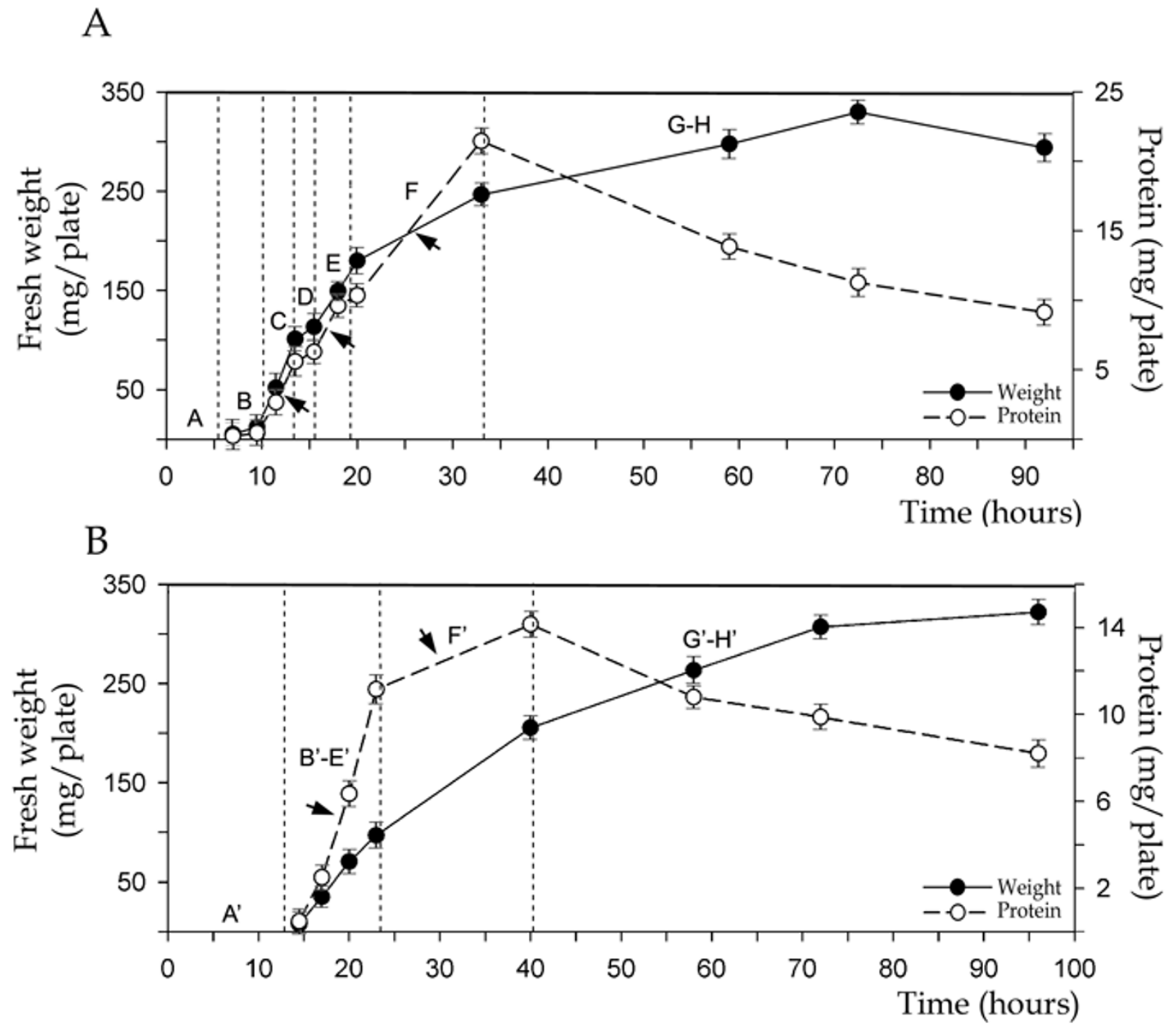

Figure 3

Growth curves (total protein and fresh weight per plate) of Streptomyces antibioticus ATCCI I89 I on surface GAE cultures developed from normal (A, I.5 $\times 10^{6}$ spores per plate) and diluted (B, $6 \times 10^{3}$ spores per plate) inocula. Arrows point to the exponential growth stages. Developmental phases $\left(A-H\right.$ and $\left.A^{\prime}-H^{\prime}\right)$ are reflected in the curve. Error bars indicate \pm SD.

Effect of the inoculum: density determines the presence of circles during development

The experiments described above were performed with a sufficient density of spore inocula as to produce rapid, confluent growth on the plates and represent the conditions often encountered in the laboratory during any given morphological and physiological analysis of Streptomyces [26], see Methods]. When a highly diluted inoculum was used $\left(6 \times 10^{3}\right.$ spores per plate), a significant delay was observed; sporulation was less efficient, and circles did not form (Figure 2). The phases indicated with apostrophes to distinguish them from their equivalents with the undiluted inoculum) are described below.

Phase A' (0-15 hours) corresponds to the germination and first death round similar to the corresponding cycle phase obtained with a normal inoculum. The main difference encountered with respect to the normal inoculum is the higher proportion of spores that present a viable, non-variegated germ tube (Phase A; not shown). 

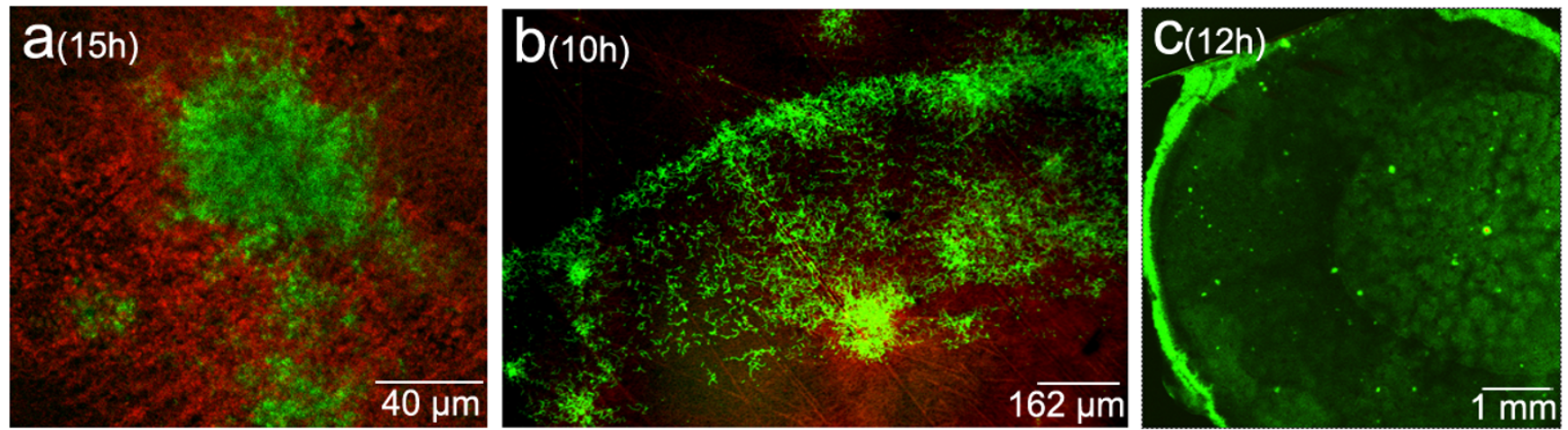

\section{Figure 4}

Longitudinal sections of different Streptomyces strains growing in confluent surface cultures. (a) S. coelicolor A3(2). (b) S. glaucescens ETH22794. (c) S. antibioticus ETH745I. Culture times are indicated. Samples were stained with SYTO 9 and propidium iodide.

However, when density increased and the hyphae touch one another, all of them die giving rise to the variegated appearance. Figure 2A' (10 hours) shows a group of hyphae that are beginning to lose their uniform green fluorescence (arrow), developing the variegated appearance which results from the alternating live and dead segments within the same hypha (on the right of the picture). This is indicative of the change in membrane permeability revealed by the propidium iodide that stains the dying segments red (see Methods).

Phase B'-E' (15-24 hours). No late-germinating spores remain at these time points (see Discussion). The live segments of the initial mycelium begin to develop in relatively regular, fast-growing islands. In this case, a unique, exponential growth phase is observed, unlike the various growth phases seen with normal inoculum. Figure $2 \mathrm{~B}^{\prime}-\mathrm{E}^{\prime}$ ( 20 hours) is a superficial view of an island formed by the emerging live mycelium.

Phase F' (24-40 hours) corresponds to the growth phase of the mycelium located between the islands. It is comparable to the mycelial growth between the circles described in Phase F of the developmental cycle in the presence of normal inoculum (Figures $2 \mathrm{~F}_{1}{ }_{1}$ and $2 \mathrm{~F}_{2}{ }_{2}, 24$ hours)

Phase G'-H' (40-96 hours) is the sporulation phase. The mycelial distribution in islands that began in Phases B'-E' is maintained. Like the circles, the islands also delimit the areas with a higher spore density (Figure $2 \mathrm{G}^{\prime}-\mathrm{H}^{\prime}, 80$ hours). Figure $2 \mathrm{G}^{\prime}-\mathrm{H}^{\prime}$ ( 80 hours) presents a detail of the surface revealing the spore chains.

In conclusion, the main difference observed with the diluted inoculum is the absence of circles originating from the delayed spore germination. The relevance of this will be discussed later.

\section{Longitudinal heterogeneity determines multiphase growth curves}

The methodological approach applied in this study eneabled us to obtain a high degree of synchronization in the plate cultures, thereby making it possible for us to analyze the relationships between growth rates and successive developmental steps. Figure 3 shows the growth curves (total protein and fresh weight per plate) obtained using normal or diluted inocula. The different phases described (see above) are indicated on the graph. Note the existence of three distinct stages of exponential growth in the normal cycle (indicated with arrows on the normal inoculum graph, Figure 3A), separated by two phases of temporary growth arrest (see the protein curve in Figure 3A). The second temporary growth arrest is not seen in the weight curve, probably because this parameter is less sensitive. The three waves of exponential growth correspond to Phase C (growth of the hyphae forming the edge of the circles), Phase E (rapid growth of the live hyphae from the center of the circles outwards) and Phase $\mathrm{F}$ (growth of the mycelium between the circles). The growth arrest periods correspond to Phase B (slow formation of the mycelial rings from late-germinating spores), Phase D (slow growth induction in the live segments of the dead hyphae located in the center of the circles) and the latter stage of Phase E (slow growth induction of the live segments of the dead hyphae located between the larger circles). Total protein per plate declines during the second death round, whereas weight continues to increase at a slower rate. This may be due to the accumulation of reserve compounds (glycogen and trehalose) within the cells [27]. Figure 3B shows the growth curves (fresh weight and total protein) 

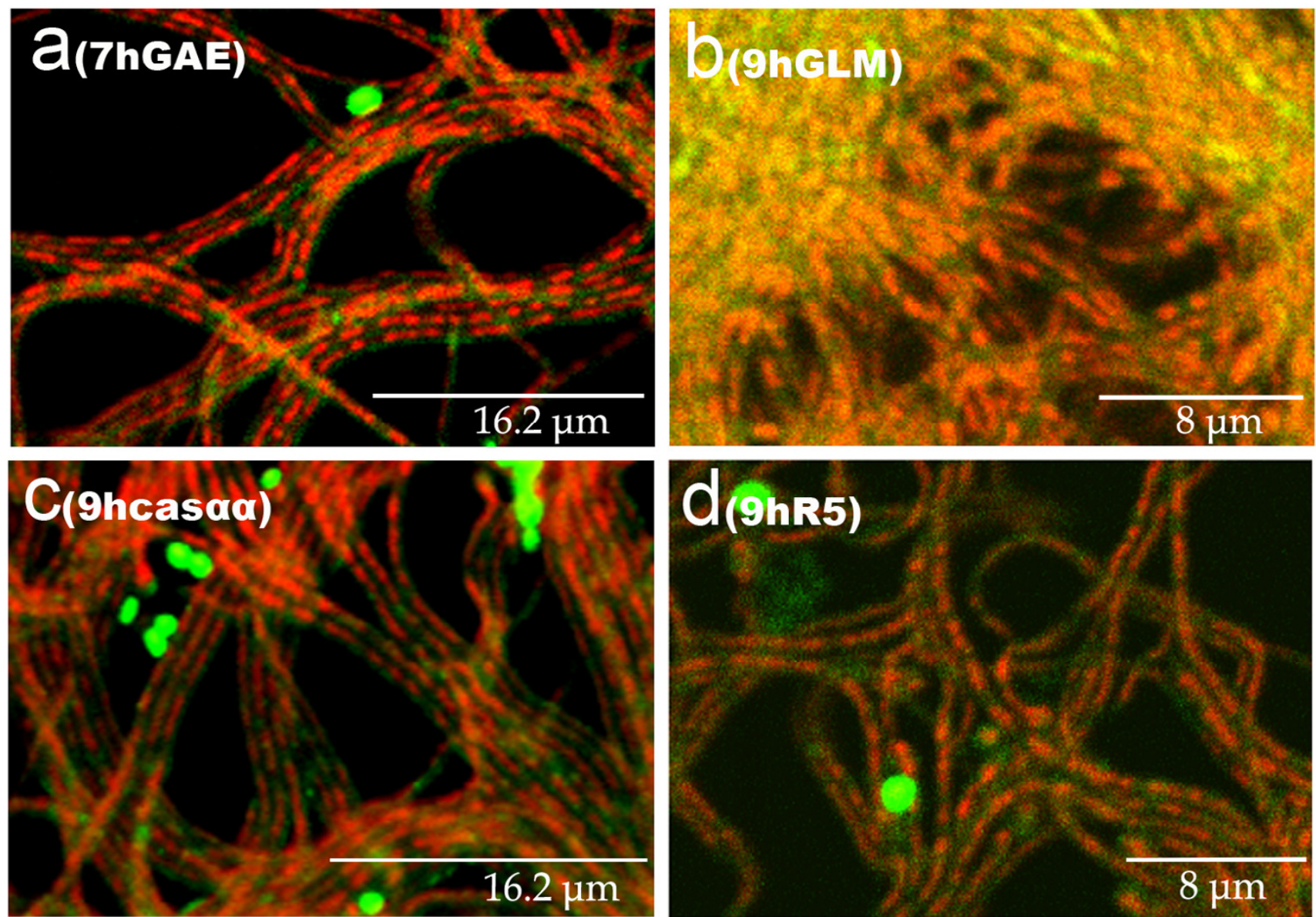

\section{Figure 5}

Cross sections of S. antibioticus ATCCI I89I cultures in different solid media. Developmental time points and culture media are indicated. Cas $\alpha \alpha$ is GAE plus $2 \%$ casamino acids. Samples were stained with SYTO 9 and propidium iodide.

for diluted inoculum $\left(6 \times 10^{3}\right.$ spores per plate). In these conditions, only two periods of exponential growth are observed, coinciding with mycelial development in the form of islands and the development of mycelium located between the islands, respectively (Figure 3B, arrows). Hence, there is a close correlation between morphological phases and growth curves. These developmental dynamics lead to the appearance of a layer of mycelium arranged in circles measuring approximately $0.5 \mathrm{~cm}$ or in islands measuring $0.9 \mathrm{~mm}$ separated by areas with a lower density of mycelium.

\section{The first death round and longitudinal heterogeneity are general phenomena in surface cultures of the Streptomyces genus}

The Streptomyces strains were grown in media in which the complete life cycle takes place with abundant sporulation (see Methods). All the Streptomyces species/strains analysed in our laboratory (with the exception of $S$. coelicolor A3(2); see below), present the pattern reported for $S$. antibioticus ATCC11891: circles when dense inocula are used and islands in the presence of highly diluted inocula. The only differences between the developmental cycles studied are the times required to reach the different phases and the diameter of the circles. Figures $4 \mathrm{~b}$ and $4 \mathrm{c}$ show Streptomyces glaucescens ETH22794 and Streptomyces. antibioticus ETH7451 circles (compare with Figures 1C and $1 \mathrm{E})$. As already mentioned, $S$. coelicolor $\mathrm{A} 3(2)$ is the only exception to the described pattern: circles are not formed in any inoculum condition (concentrated or diluted) and development always occurs in the form of islands (Figure 4a). 
In media in which S. antibioticus ATCC11891 do not sporulate (GYM, GAE plus 2\% casamino acids and R5; see Methods), the first death round and the variegated hyphae are also present (Figure 5) and longitudinal heterogeneity is observed (not shown). However, the second death round and sporulation are absent.

\section{Discussion}

All studies to date describing the differentiation and developmental cycle of Streptomyces refer to a completely live, viable substrate mycelium that grows inside the culture medium from which a reproductive (aerial) mycelium emerges after a massive death round. The most superficial part of the aerial mycelium forms spores by septating into chains of uninucleate compartments, whereas the non-sporulating aerial mycelium and the substrate mycelium eventually die [6,22-24]. In accordance with this model, the substrate mycelium in Streptomyces antibioticus ATCC11891 is the mycelium formed from spores and developed until approximately 35 hours of cultivation at $28-30^{\circ} \mathrm{C}$. The formation of aerial mycelium commences at this point and at approximately 60 hours of cultivation, the sporulation process begins $[22,28]$. The three mycelial types (substrate, aerial and sporulated aerial) exist simultaneously at certain times. Again, according to this model, this cycle is present in all Streptomyces species, albeit with certain temporal variations.

In this work we have analysed the developmental cycle of Streptomyces in three spatial dimensions, the transverse and longitudinal axes, a perspective we believe is essential to understanding the developmental cycle. The developmental features of S. antibioticus ATCC11891 on confluent surface cultures are summarized in Figure 6. An early death round takes place affecting the young substrate hyphae, which to date, has not been described. This death process occurs in a remarkably symmetrical form: live and dead segments alternate within the same hyphae in a very regular pattern (Figure 1A, 6 hours). Subsequently, the remaining mycelium grow in successive waves, creating longitudinal heterogeneity of the bacterium on the plate surface. In all prior studies of the Streptomyces developmental cycle in solid media, it is implicitly assumed that the cultures are homogeneous all over the plate surfaces; hence, the point at which the plate is analysed would not be critical. The only heterogeneity present would be in the transverse plane; that is from the bottom up. Our data clearly demonstrate that substrate and aerial mycelial growth is heterogeneous and orderly, forming a remarkable pattern of circles and islands. This finding suggests that diffusible signals are involved in their induction.

As occurs with other bacteria, Streptomyces cultures are niches where quorum-sensing phenomena take place
[29], particularly in the relatively crowded conditions normally employed in the laboratory for growing surface cultures. Many signals of this type have been reported in the Streptomyces genus [30]. The absence of circles in diluted inoculum conditions might also be related to the lack of appropriate signals. The circles are formed by spores that germinate at a later time, under the influence of signals that determine their growth as viable mycelium that does not die at the earlier time points (see above). Bearing in mind that the circles initially form at very early time points (Figures 1 and 6), it can be speculated that at low inoculum densities, the hypothetical signals do not accumulate enough to induce these structures. Another simple alternative explanation concerns the origin of the circles. Given that they are initially comprised of late-germinating spores that are logically less common in highly diluted inocula, it can be hypothesized that when the mycelial layer is dense enough to produce the signals, no late-germinating spores remain and hence, the edge of the circles is not formed. The phenomena described here are clearly different from other surface phenotypes, such as the previously described Streptomyces "pocks" [31]. The pocks are caused by the presence of conjugative plasmids and their appearance is due to the slower rate of mycelial growth at these points $[26,31]$. The opposite happens with the circles described in the present work, which are made up of a high mycelial density (see above). Some authors have described the existence of circular areas of unknown origin, which were "not genuine pocks", yet were present in many Streptomyces cultures [26]. These areas probably correspond to the circles described in this paper.

Our data clearly show that longitudinal heterogeneity is at least as important as transverse heterogeneity in the development of Streptomyces surface cultures. Very different developmental phases may therefore be taking place simultaneously at two different points of the surface cultures (Figures 1 and 6). For example, in Phase E, the live mycelium inside the circles is fully developed and in the process of dying, whereas the mycelium between the circles is just beginning to grow. This is fundamental in order to properly understand and interpret the distinct stages that occur during the differentiation cycle of Streptomyces. With the exception of S. coelicolor A3(2), all the Streptomyces species analysed in our laboratory present the pattern reported for S. antibioticus ATCC11891. S. coelicolor do not form circles in any inoculum condition and instead, only present surface development in the form of islands (Figure 4). Knowing whether one specific species or strain of Streptomyces grows in "circles" or "islands" is key for the correct interpretation of the morphological/ biochemical data obtained in solid cultures, as illustrated by the growth curves (Figure 3). In media in which there is no sporulation, the first death round takes place and longitudinal heterogeneity is present; however, the sec- 


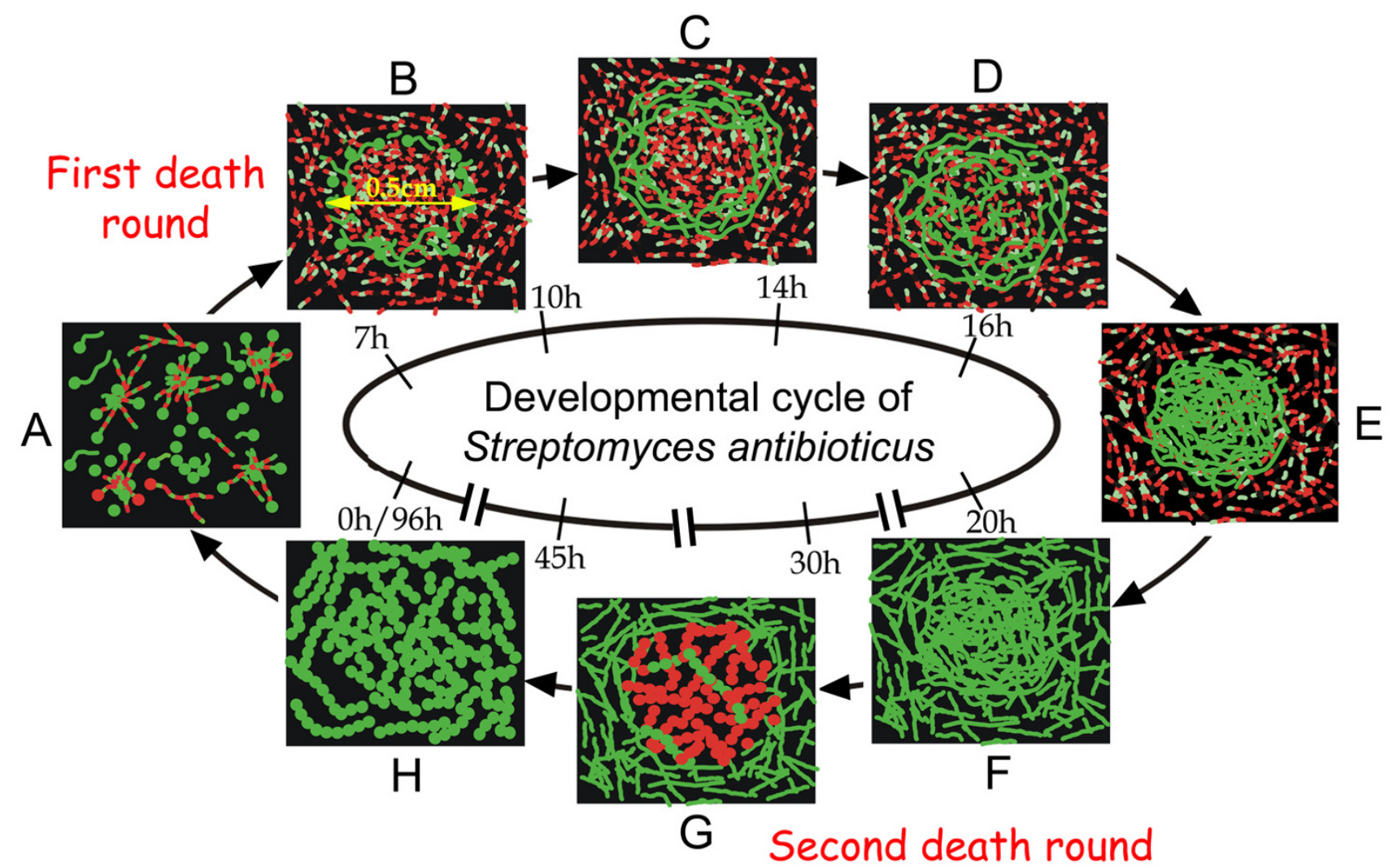

Figure 6

Model for the developmental cycle of S. antibioticus ATCCI I89I on surface GAE cultures (detailed in text). Times are indicated in hours.

ond death round does not occur. Consequently the first death round and the appearance of longitudinal heterogeneity are general events inherent to the development of Streptomyces on surface cultures. We are currently analysing the peculiarities of several Streptomyces species in different culture media, in order to integrate these into a consensus and create a reliable model of Streptomyces development on surface cultures.

A vast collection of mutant Streptomyces [32,33] will be generated in the future thanks to the new, powerful techniques currently available. Factoring the features described here into the analysis of these and other previously reported differentiation mutants of Streptomyces will greatly expand the number of potential phenotypes to be considered and hence, their corresponding genetic determinants. This in turn, will hopefully facilitate the discovery of new signal cascades in this important bacterium. Furthermore, as the differentiation of hyphae and antibi- otic production share common genetic control elements [34-36], the aspects considered above will also aid in better understanding antibiotic production in solid-state fermentation [37-39], as well as in submerged conditions [40], A. Manteca and J. Sanchez, unpublished data].

\section{Methods \\ Strains and media}

Streptomyces antibioticus ATCC11891, Streptomyces. antibioticus ETH7451, Streptomyces glaucescens ETH22794 and Streptomyces coelicolor A3(2) were the species used in this research. The microorganisms were grown in solid media in which they present a complete life cycle with abundant sporulation. S. antibioticus ATCC11891 and S. glaucescens ETH22794 were grown on GAE medium [22]; S. coelicolor A3(2) was grown on GYM medium (glucose, yeast extract, malt extract; 41). S. antibioticus was also grown on media in which it does not sporulate: GAE plus $2 \%$ casamino acids, GYM [40] and R5 [25]. The cultures were prepared 
in Petri dishes ( $8.5 \mathrm{~cm}$ diameter) as lawns on solid medium (30 ml/plate). When indicated, sterile cellophane disks were placed on the surface prior to inoculation. Under the conditions used, the differentiation processes follow a pattern similar to that of mycelium incubated directly on the culture medium [22]. Plates (with or without cellophane) were inoculated directly with $100 \mu \mathrm{l}$ of a spore suspension $\left(1.5 \times 10^{7}\right.$ viable spores/ $\mathrm{ml}$ ), followed by incubation at $30^{\circ} \mathrm{C}$. In some cases, plates were inoculated with a highly diluted spore suspension $\left(6 \times 10^{3}\right.$ spores per plate).

\section{Microscopy}

Culture samples were obtained and processed for microscopy at different incubation times, as described previously for submerged and surface-grown Streptomyces cultures $[12,13]$. Petri dishes prepared with Difco agar and inoculated as described above were used to obtain solid blocks of the agar cultures with a scalpel. These blocks were further trimmed to squares of approximately $10 \mathrm{~mm}$ in size and introduced into a hand microtome $(11 \mathrm{~mm}$ hole diameter) previously cooled to $4^{\circ} \mathrm{C}$, with the surface of growth facing sideways. Sections of about $0.3 \mathrm{~mm}$ were obtained (cross sections). To analyse longitudinal sections, we used solid cultures covered with cellophane disks: the disks were removed from the culture, cut into squares measuring approximately $1.5 \mathrm{~cm} \times 1.5 \mathrm{~cm}$ and placed on slides before staining.

The permeability assay previously described for Streptomyces was used to stain all samples [12]. This technique involves staining the cells with cell-impermeant nucleic acid stain (propidium iodide, PI) in order to detect the dead cell population of $S$. antibioticus and with SYTO 9 green fluorescent nucleic acid stain (LIVE/DEAD BacLight Bacterial Viability Kit, Molecular Probes, L-13152) to detect viable cells. The SYTO 9 green fluorescent stain labels all the cells, i.e. those with intact membranes, as well as those with damaged ones. In contrast, PI penetrates only bacteria with damaged membranes, decreasing SYTO 9 stain fluorescence when both dyes are present. Thus, in the presence of both stains, bacteria with intact cell membranes appear in fluorescent green, whereas bacteria with damaged membranes appear in red [15]. The stain mixture was prepared as per the manufacturer's instructions and was added directly on the samples on the slide. The coverslide was placed on top and after staining for at least 10 minutes in the dark, the samples were then examined under a Leica TCS-SP2-AOBS confocal laserscanning microscope at a wavelength of $488 \mathrm{~nm}$ and 568 $\mathrm{nm}$ excitation and $530 \mathrm{~nm}$ (green) or $630 \mathrm{~nm}$ (red) emission. Images were mixed using the Leica Confocal Software. In some cases, the samples were also examined in differential interference contrast mode, available with the same equipment.

\section{Streptomyces cell sampling and processing}

Cells from S. antibioticus grown on the surface of cellophane disks were scraped off with a plain spatula at different time points. Growth curves were obtained by means of weight determinations and total protein analysis. Fresh weight data (obtained without subjecting the mycelium to any drying treatment) were used, given that these data are reproducible and afford reliable determination of growth values in the early stages of the cultures when the small amount of mycelium per plate at these time points makes the use of other alternative approaches difficult. Determinations were repeated a minimum of three times. Total protein was analysed in the collected mycelium as follows: the weighted mycelium was resuspended in buffer A (Tris-HCl $20 \mathrm{mM} \mathrm{pH} \mathrm{8,} \mathrm{EDTA} 1 \mathrm{mM}, \beta$-mercaptoetanol 7 $\mathrm{mM}$ and PMSF $0.5 \mathrm{mM}$ ), maintaining a constant ratio between fresh weight of mycelium and volume of buffer A ( $65 \mathrm{mg}$ mycelium/ml buffer A). The suspension was ruptured in an MSE soniprep 150, in 6 cycles of 10 seconds, on ice, after which the samples were centrifuged at 10000 r.p.m. in an Eppendorf microcentrifuge for $30 \mathrm{~min}$ at $4{ }^{\circ} \mathrm{C}$ and the protein was determined by the Lowry assay [42].

\section{List of abbreviations}

CLSM, confocal laser-scanning fluorescence microscopy; PI, propidium iodide.

\section{Authors' contributions}

AM performed all microscopic and biochemical analyses. MF participated in some of the experiments and in the initial study design. JS conceived the study, participated in its design, and coordinated and drafted the manuscript. All authors read and approved the final manuscript.

\section{Acknowledgements}

We wish to thank Angel Martinez Nistal, Image Processing and Analysis Service of the University of Oviedo for his indispensable assistance with the confocal microscope and Priscilla A. Chase for revising the text. This research was funded by grant BIO2000-0577 from the DGI, Subdirección General de Proyectos de Investigación, MCYT, Spain.

\section{References}

I. Moran MA, Rutherford LT, Hodson RE: Evidence for indigenous Streptomyces populations in a marine environment determined with a I6S rRNA probe. Appl Environ Microbiol I995, 6 I:3695-3700.

2. Schatz A, Bugie E, Waksman SA: Streptomycin, a substance exhibiting antibiotic activity against gram-negative and gram-positive bacteria. Proc Soc Expt/Biol Med 1944, 55:66-69.

3. Wasksman SA, Woodruff HB: The soil as a source of microorganisms antagonistic to disease-producing bacteria. J Bacteriol 1940, 40:581-600.

4. Paradkar A, Trefzer A, Chakraburtty R, Stassi D: Streptomyces genetics: a genomic perspective. Crit Rev Biotechnol 2003, 23:1-27.

5. Waksman SA: The Genus Streptomyces. In The Actinomycetes. A Summary of Current Knowledge Volume Chapter 9. The Ronald Press Co. NY; 1967

6. Hodgson DA: Differentiation in Actinomycetes. In Prokaryotic Structure and Function: A New Perspective. Society for General Microbiology 
Symposium Volume 47. Edited by: Mohan S, Dow C, Cole JA. Cambridge University Press, Cambridge; 1992:407-440.

7. Hopwood DA, Glauert AM: Electron microscope observations on the surface structures of Streptomyces violaceoruber. J Gen Microbiol I96I, 26:325-30.

8. Granozzi C, Billeta R, Passantino R, Sollazzo M, Puglia AM: A breakdown in macromolecular synthesis preceding differentiation in Streptomyces coelicolor A3(2). J Gen Microbiol 1990 136:713-716.

9. Claessen D, Rink R, de Jong W, Siebring J, de Vreugd P, Boersma FG, Dijkhuizen L, Wosten HA: A novel class of secreted hydrophobic proteins is involved in aerial hyphae formation in Streptomyces coelicolor by forming amyloid-like fibrils. Genes Dev 2003, I7:1714-1726.

10. Elliot MA, Karoonuthaisiri N, Huang J, Bibb MJ, Cohen SM, Kao CM, Buttner MJ: The chaplins: a family of hydrophobic cell-surface proteins involved in aerial mycelium formation in Streptomyces coelicolor. Genes Dev 2003, I7:1727-1740.

II. Wildermuth $H$, Wehrli $E$, Horne RW: The surface structure of spores and aerial mycelium in Streptomyces coelicolor. J Ultrastruct Res 1971, 35: 168-80.

12. Fernandez M, Sanchez J: Nuclease activities and cell death processes associated with the development on surface cultures of Streptomyces antibioticus ETH745I. Microbiology 2002, I 48:405-4I 2 .

13. Fernandez M, Sanchez J: Viability staining and terminal deoxyribonucleotide transferase-mediated dUTP nick end labeling of the mycelium in submerged cultures of Streptomyces antibioticus ETH745 I. J Microbiol Methods 200I, 47:293-298.

14. Manteca A, Fernandez M, Sanchez J: Cytological and Biochemical Analysis of two Lytic programmed Cellular Dismantling Rounds accompanying the development of Streptomyces antibioticus in Surface Cultures. Res Microbiol in press.

15. Haugland RP: Nucleic acid detection and genomics technology. In Handbook of Fluorescent Probes and Research Chemicals Volume Chapter 8. Ninth edition. Edited by: Gregory J. Molecular Probes, Inc Eugene, OR; 2002.

16. Bunthof CJ, van Schalkwijk S, Meijer W, Abee T, Hugenholtz J: Fluorescent method for monitoring cheese starter permeabilization and lysis. Appl Environ Microbiol 200I, 67:4264-7I.

17. Bunthof CJ, Sabina van den Braak S, Breeuwer P, Rombouts FM, Abee $\mathrm{T}$ : Rapid Fluorescence Assessment of the Viability of Stressed Lactococcus lactis. Appl Envir Microbiol 1999, 65:368I-3689.

18. Lloyd $D$, Hayes $A$ : Vigour, vitality and viability of microorganisms. FEMS Microbiol Lett 1995, I33:1-7.

19. Miller JS, Quarles JM: Flow cytometric identification of microorganisms by dual staining with FITC and PI. Cytometry 1990, I I:667-675.

20. Darzynkiewicz Z, Bruno S, Del Bino G, Gorczyca W, Hotz MA, Lassota $P$, Traganos F: Features of apoptotic cells measured by flow cytometry. Cytometry 1992, 13:795-808.

21. Sebastine IM, Stocks PW, Cox PW, Thomas CR: Characterization of percentage viability of Streptomyces clavuligerus using image analysis. Biotechnol Tech 1999, 13:419-423.

22. Mendez C, Braña AF, Manzanal MB, Hardisson C: Role of substrate mycelium in colony development in Streptomyces. Can J Microbiol 1985, $31: 446-450$.

23. Kalakoutskii LV, and Agre NS: Comparative aspects of development and differentiation in actinomycetes. Bacteriol Rev 1976, 40:469-524.

24. Wildermuth $\mathrm{H}$ : Development and organization of the aerial mycelium in Streptomyces coelicolor. J Gen Microbiol 1970, 60:43-50.

25. Chater KF: Aspects of multicellular differentiation in Streptomyces coelicolor A3(2). In Genetics and Molecular Biology of Industrial Microorganisms Edited by: Hershberger CL, Queener SW, Hegeman G. American Society for Microbiology, Washington, DC; 1989:99-107.

26. Kieser T, Bibb MJ, Buttner MJ, Chater KF, Hopwood DA: Practical Streptomyces Genetics. The John Innes Foundation, Norwich, England; 2000.

27. Braña AF, Mendez C, Diaz LA, Manzanal MB, Hardisson C: Glycogen and trehalose accumulation during colony development in Streptomyces antibioticus. J Gen Microbiol 1986, I32:|319-1326.
28. Miguelez EM, Hardisson C, Manzanal MB: Hyphal death during colony development in Streptomyces antibioticus : morphological evidence for the existence of a process of cell deletion equivalent to apoptosis in a multicellular prokaryote. J Cell Biol 1999, 145:515-525

29. Bayles KW: Are the molecular strategies that control apoptosis conserved in bacteria? Trends Microbiol 2003, I I:306-3 II.

30. Bibb MJ: Regulation of secondary metabolism in Streptomycetes. Curr Opin Microbiol 2005, 8:208-2I5.

3I. Pettis GS, Ward N, Schully KL: Expression characteristics of the transfer-related kilB gene product of Streptomyces plasmid pIJIOI: implications for the plasmid spread function. Bacteriol 200I, I 83: I339-1345.

32. Gehring AM, Nodwell JR, Beverley SM, Losick R: Genomewide insertional mutagenesis in Streptomyces coelicolor reveals additional genes involved in morphological differentiation. Proc Natl Acad Sci U S A 2000, 97:9642-9647.

33. Sprusansky O, Zhou L, Jordan S, White J, Westpheling J: Identification of three new genes involved in morphogenesis and antibiotic production in Streptomyces coelicolor. J Bacteriol 2003, 185:6|47-6I57.

34. Champness WC, Chater KF: Regulation and integration of antibiotic production and morphological differentiation in Streptomyces sp. In Regulation of Bacterial Development Edited by: Piggot PJ, Morgan CP, Youngman P. American Society for Microbiology, Washington, DC; 1994:6I-93.

35. Chater KF, Bibb MJ: Regulation of bacterial antibiotic production. In Biotechnology Products of Secondary Metabolism Volume 7. Edited by: Kleinkauf H, v Dören H. VCH Press, Weinheim, Germany; 1997:57-105.

36. Horinouchi S, Ohnishi Y, Kang DK: The A-factor regulatory cascade and CAMP in the regulation of physiological and morphological development in Streptomyces griseus. J Ind Microbiol Biotechnol 200I, 27:177-182.

37. Ellaiah P, Srinivasulu B, Adinarayana K: Optimisation studies on neomycin production by a mutant strain of Streptomyces marinensis in solid state fermentation. Process Biochem 2004, 39: $1331-1339$.

38. Jermini MF, Demain AL: Solid state fermentation for cephalosporin production by Streptomyces clavuligerus and Cephalosporium acremonium. Experientia 1989, 45: I06I-1065.

39. Kota KP, Sridhar P: Solid state cultivation of Streptomyces clavuligerus for cephamycin C production. Process Biochem 1999, 34:325-328

40. Stocks SM, Thomas CR: Viability, strength, and fragmentation of Saccharopolyspora erythraea in submerged fermentation. Biotechnol Bioeng 200I, 75:702-709.

4I. Novella IS, Barbes C, Sanchez J: Sporulation of Streptomyces antibioticus ETH 745 I in submerged culture. Can J Microbiol 1992, 38:769-773.

42. Lowry OH, Rosenbrough NJ, Farr AL, Randall RJ: Protein measurement with the Folin phenol reagent. J Biol Chem I95I, 193:265-275.

Publish with Bio Med Central and every scientist can read your work free of charge

"BioMed Central will be the most significant development for disseminating the results of biomedical research in our lifetime. "

Sir Paul Nurse, Cancer Research UK

Your research papers will be:

- available free of charge to the entire biomedical community

- peer reviewed and published immediately upon acceptance

- cited in PubMed and archived on PubMed Central

- yours - you keep the copyright
BioMedcentral 